\title{
Creating Video Content for Oculus Rift Scriptwriting for $360^{\circ}$ interactive video productions
}

\author{
Mirjam Vosmeer and Ben Schouten \\ Interaction \& Games Lab, \\ Amsterdam University of Applied Sciences \\ Postbus 1025, 1000 BA Amsterdam, The Netherlands \\ Phone: 0618848410 \\ m.s.vosmeer@hva.nl, b.a.m.schouten@hva.nl
}

\begin{abstract}
In this workshop, we will first discuss previous experiences with producing and/or watching video content for Oculus Rift, or other $360^{\circ}$ video devices. After determining the challenges and possibilities, we will works towards developing concepts for settings and stories for this particular medium.
\end{abstract}

Keywords. interactive narrative, digital storytelling, scriptwriting, content development, Oculus Rift

\section{Introduction/workshop background}

Oculus is awesome for games, but it's the future of movies, was the headline of an article that was published in January 2014 in technology and lifestyle magazine Wired (Watercutter, 2014). After Facebook paid 2 billion dollar to take over the company Oculus VR in March 2014 (Solomon, 2014), the headset Oculus Rift became world famous practically overnight. In the past year, researchers and developers who had gotten hold of the first series of developer kits have been presenting their latest findings on technology festivals and conferences all over the world, and discussing the challenges they encountered when producing 360 degrees content for this device (for instance, Vosmeer and Schouten, 2014). Especially developers who explore the possibilities of creating real life video content (instead of VR content), find themselves probing a new field of media production that offers characteristics of video games on one hand, but on the other hand feels like a movie in which the viewer is watching a scene that is displayed all around him or her. 
While using Oculus Rift, the viewer has the sense of literally being in the center of the scene. Because the footage has been recorded in 360 degrees, the viewer has the strange sense of being present in the movie, almost as if he or she is playing a part in it, instead of watching from the outside. This relates directly to the theoretical concept of 'presence', which is defined as 'being in the story world' (Roth et al, 2012). The perspective is actually more related to the position of a video game player than that of a movie watcher. After all, gamers are used to being able to look all around in the game world, in order to explore the surroundings and detect enemies or other game elements. When watching a movie, viewers are presented with the one frame that has been chosen by the director to present the story through. One of the challenges that producers face is that they need to find ways to tell stories for surround video content that suit the sense of presence that users experience. One solution to this problem is adding voice-over narration: the voice of someone talking who is not visible on the screen (Kozloff, 1988).

In our first study, we have experimented with different voice over perspectives that would best suit the immersive qualities that $360^{\circ}$ video content offers its users. It turned out that a second person perspective gave the audience the best sense of presence: of actually being a part of the narrative that was presented.

Our second study focused on creating an interactive story that could be produced for Oculus Rift. We developed a concept, explored different ways of interactive storytelling, and eventually produced a short interactive drama for Oculus Rift that was presented in Amsterdam in January 2015.

The 360' point of view implies several other difficulties for the producer. Firstly, it is not possible to work with a traditional film set in which a scene may be recorded. As the camera records the world in 360', everything around the system will be seen. This problem also applies for the floor of the set, and the ceiling. As these angles will also be available for the future viewer, both the floor and the ceiling of the set need to be fully presentable, which means for instance that it is not possible to make use of elaborate lighting systems (Vosmeer and Schouten, 2014). Another issue involves camera movement: while the viewer takes the point of view of the camera, and is able to look around in the scene, he or she is not able to move through the space in which the scene was filmed. The only possible movement is the one that was recorded when shooting the scene.

The camera position within a setup like this also has profound impact on the way that stories may be told. A scene that is written with the intention to be watched from 360', may take different viewer perspectives into account. For instance, the story could be told in such a way that the camera is not part of the narrative, but just registers the scene like a fly on the wall. Another option however, would be to include the viewer position within the story, and have characters react towards the camera as if it is another character participating in the scene. Experiments like these, in which actors react to a camera, are of course known within traditional cinema as well. With this new technology, however, experiments with new ways of storytelling are imaginable in which the option of the viewer to look around within the scene - and for instance 
discover pieces of information and new interpretations - could be essential to the way a plot unfolds.

Another interesting possibility that this kind of media production offers, is the use of so called hot-spots: it is possible to insert interactive points in the video that can be activated by the user by focusing his or her gaze on it for several moments (this can be compared to clicking a link or touching a screen). These interactive points can be used to activate the next part of the story. For instance: in our Oculus Rift video 'A Perfect Party', the user was told by the voice over that he was hosting a party, but that it would be nice to have some music. If the user than fixed his gaze on the DJ for a few seconds, the DJ would step forward and start playing music, which would result in the guests starting to dance.

\section{Workshop}

In this half-day workshop, we will first present a short overview of our previous studies into content creation for Oculus Rift, and explain the challenges and possibilities that we came across. Secondly, we will discuss the attendants' own earlier experiences with $360^{\circ}$ video content, and invite them to reflect on the narrative aspects that they have encountered there. In the third part of the workshop, attendants will be asked to think of possible settings in which an Oculus Rift narrative could be placed, taking into account the production boundaries that were discussed earlier. During the fourth and last part of the workshop, we will discuss the settings, think of story concepts that might be written for these particular settings, and consider whether using a voice over could add useful extra information to the scene. Note that the focus will be on developing concepts and narratives, rather than on dealing with technical issues.

\section{Workshop outcomes}

During this workshop, we hope to develop new concepts for settings and stories that can be produced for Oculus Rift. After attending the workshop, the participants will have gained new insights into the production challenges and narrative possibilities of producing $360^{\circ}$ interactive stories.

\section{$4 \quad$ Participants}

I think that a maximum of 12 participants would be a good number for this workshop, but I have no specific names yet. 


\section{$5 \quad$ CV Information}

Mirjam Vosmeer is coordinator of the Interaction \& Games Lab at the Amsterdam University of Applied Sciences. She has a $\mathrm{PhD}$ in communication science and an academic background in game studies, psychology and media entertainment. Her further professional background includes television production, scriptwriting and game concept development. She is project manager of Interactive Cinema, a collaboration between the Netherlands Film Academy and the Amsterdam University of Applied Sciences. For this project, she has conducted several experiments into interactive storytelling for $360^{\circ}$ video content. Ben Schouten is professor Playful Interaction at Eindhoven University and lector Play and Civic Media at the Amsterdam University of Applied Sciences

\section{Acknowledgement}

This workshop proposal is part of the Interactive Cinema project, within the Amsterdam Creative Industries Network. The Interactive Cinema project is a collaboration between the Netherlands Film Academy and the Amsterdam University of Applied Sciences. The pilot for this workshop has been presented on March 27 (2015), for a group of screenwriting students who have graduated from the Dutch Film Academy.

\section{$7 \quad$ References}

1. Kozloff, S. Invisible Storytellers. University of California Press: Berkeley, 1988.

2. Roth, C., Klimmt, C., Vermeulen, I.E., and Vorderer, P. "The experience of interactivestorytelling: Comparing "Fahrenheit" with "Façade"," in Proceedings of ICEC 2011, IFIP, pp. 13-21.

3. Solomon, B. "Facebook buys Oculus virtual reality gaming startup for 2 billion." Forbes 2014. Available at

http://www.forbes.com/sites/briansolomon/2014/03/25/facebook-buys-oculus-virtualreality-gaming-startup-for-2-billion/ (retrieved March, 2015)

4. Vosmeer, M. and Schouten, B. "Interactive Cinema: Engagement and interaction." In proceedings of ICIDS 2014. Springer International Publishing, pp. 140-147.

5. Watercutter, A. Oculus Is Awesome for Games, But It's the Future of Movies. http://www.wired.com/2014/01/oculus-movies/ (2014) (retrieved March, 2015) 